\title{
EVALUACIÓN DEL PAPEL DE LOS INTERCAMBIOS DE DOCENTES DE ESTUDIOS GENERALES PARA LA FORMACIÓN INTEGRAL DEL ESTUDIANTE
}

\author{
Evaluation of the role of the exchanges of teachers \\ of General Studies for the integral formation of the student
}

\author{
Waldemiro Vélez Cardona ${ }^{1} \bullet$ María Elena Córdoba ${ }^{2}$
}

Recibido: 13-9-2016 • Aprobado: 15-1-2017

\begin{abstract}
Resumen
El artículo evalúa el impacto que tuvo el programa de movilidad docente en el que participaron el Instituto Tecnológico de Santo Domingo (INTEC), la Pontificia Universidad Católica del Perú (PUCP) y la Universidad de Puerto Rico, Recinto de Río Piedras), realizado en el período 2014-2015. El programa permitió conocer las características de esta herramienta de formación en las tres universidades participantes, además de su filosofía y sus alcances. El estudio también abordó la repercusión de los intercambios de docentes de Estudios Generales respecto al desarrollo de la sensibilidad intercultural y las estrategias docentes que se utilizan en cada universidad para trabajar contenidos similares o problemáticas particulares. La investigación se subdividió en dos etapas, cada una de las cuales aportó sus propios resultados. En esta, se evalúa la aportación de los intercambios docentes, por medio de su incidencia en la capacitación de los profesores de estudios generales, en algunas dimensiones destacadas de la formación integral del estudiante. En primer lugar, se hace un estudio comparativo de las ejecutorias docentes, por medio de una herramienta diseñada a esos efectos. En segundo lugar, se recoge la impresión de los estudiantes, por medio de dos cuestionarios (uno antes de la movilidad del docente y otro después), en los que incluimos las variables que son fundamentales para dicho proceso.
\end{abstract}

Palabras clave: Estudios Generales; formación integral; movilidad docente; desarrollo del profesorado: interculturalidad.

1. Profesor de la Universidad de Puerto Rico, Puerto Rico. Correo electrónico: waldemirov@hotmail.com

\begin{abstract}
The article evaluates the impact that the teacher's mobility program had with the participation of the Instituto Tecnológico de Santo Domingo (INTEC), the Pontificia Universidad Catolica del Peru (PUCP) and the Universidad de Puerto Rico (Río Piedras campus), which took place during the year 2014-2015, in the integral students' formation. The program allowed to know the characteristics of this training tool within the three participating universities, as well as its philosophy and its scope. The study also addressed the impact of the general study teachers' exchanges concerning the intercultural sensitivity and teaching strategies' development used in each University to work similar content or particular problems. The research was subdivided into two stages, each of which contributed with their own results. This assesses the contribution of teaching exchanges, through its impact on the general study teachers' training, in some important dimensions of the integral students' formation. First of all, a comparative study of the teaching execution is performed, using a designed tool for this purposes. Second, the students' impression is gathered, through two questionnaires (one before the teacher's mobility and another after), in which we include the fundamental variables for this process.
\end{abstract}

Keywords: General Studies; integral formation; faculty mobility; faculty development; interculturality.

2. Profesora investigadora del Instituto Tecnológico de Santo Domingo (INTEC), República Dominicana. Correo electrónico: maria.cordoba@intec.edu.do 


\section{Introducción}

Este artículo corresponde a la entrega final de la segunda etapa de un estudio que comenzó en 2014 sobre los programas de Movilidad Docente en Estudios Generales. La misma se realizó en tres instituciones universitarias (Instituto Tecnológico de Santo Domingo, Pontificia Universidad Católica del Perú y Universidad de Puerto Rico, Recinto de Río Piedras). En la primera etapa se pudo apreciar cómo las diferencias culturales de las instituciones y países participantes en el estudio, así como el impacto que estaba teniendo el diseńo realizado para las estancias de cada docente y posteriores actividades al regresar a su universidad, hacían del programa un proyecto esperanzador para lograr el propósito esperado. Partimos de un supuesto: si impactábamos favorablemente en el docente, su práctica se vería enriquecida también y ello repercutiría en la formación integral del estudiantado.

Uno de los aportes significativos constatado consistió en el incremento de la reflexividad de los docentes sobre su práctica educativa, al comprobar las diferencias idiosincráticas y culturales, tanto en teoría, filosofía educativa, y prácticas concretas en las tres Instituciones que formaron parte del estudio. De la información aportada por los docentes participantes se desprende el interés por desarrollar colaboraciones interinstitucionales, además de observar cómo se han venido implantando en la práctica los Estudios Generales como importante componente de la docencia universitaria en el siglo Xxi, lo que será de gran ayuda al momento de proponer cambios curriculares que fortalezcan la formación integral del estudiantado.

Con el pasar del tiempo, los cambios y los avances en la tecnología, las instituciones de educación superior se han visto en la necesidad de cambiar sus procesos de enseñanza-aprendizaje; esto ha implicado la transición de un enfoque orientado hacia la formación académica a la formación integral. Esta formación integral sugiere la "participación activa del estudiante en la construcción de conocimiento y se privilegia el pensamiento crítico y reflexivo sobre la mera acumulación de información mediante procesos memorísticos repetitivos" (Quero, 2008).

Los programas de movilidad profesoral en las Instituciones de Educación Superior han cobrado más sentido en la realidad de un mundo globalizado, por el hecho de aportar tanto a los docentes como a las instituciones participantes, una visión mucho más amplia para la efectividad de su función primordial que es la generación de conocimientos. Paulatinamente se han convertido en un medio de capacitación docente que permite crear un arsenal de estrategias para la formación integral de los estudiantes, dentro de los Estudios Generales, partiendo de las experiencias interculturales y el enriquecimiento profesional subsecuente.

A través de esta investigación se pudo comprobar que se cumple la hipótesis de trabajo que nos planteamos al inicio de este estudio en su primera etapa y que dice: La participación en los programas de movilidad docente, particularmente los que se desarrollan en el ámbito de los estudios generales, tienen un impacto positivo y medible en el desarrollo de destrezas y actitudes de los estudiantes que conducen a su formación integral.

En la primera etapa de esta investigación se aplicaron diversos instrumentos que posibilitaron comparar las particularidades de las opciones de estudios generales en cada institución.

Para la segunda etapa del estudio, se programó la aplicación de un pos-test para su contrastación con los resultados del pre-test aplicado en la primera etapa y se realizó una entrevista a cada docente participante. La parte final de esta segunda etapa consistió en la evaluación directa del impacto del Programa de Movilidad en la práctica docente de los participantes. Para ello se aplicaron autoevaluaciones a los docentes, donde estos identificaron la presencia o ausencia de diferentes elementos de la formación 
integral en su práctica docente. La autoevaluación se realizó antes y después de la participación en el Programa de Movilidad, como forma de comparar si existieron cambios. Además, se hicieron observaciones estructuradas de las clases de los docentes participantes, utilizando como guía el mismo instrumento que se utilizó para la autoevaluación. Las observaciones, al igual que las autoevaluaciones, se hicieron antes y después de la participación en el programa.

Se elaboró un pre-test/post-test que fue aplicado a los grupos de estudiantes de los docentes que colaboraron en el programa de Movilidad, antes y después de su participación. Con este instrumento se evaluó la percepción del estudiantado respecto a la presencia de elementos conducentes a la formación integral en las prácticas de los docentes. A través de las diferentes preguntas los estudiantes establecieron en qué medida el docente cumplía con el criterio evaluado. A partir de lo anterior, fue posible comparar las diferencias de los resultados obtenidos antes y después del programa.

\section{Programas de Movilidad Docente, Estudios Generales y Formación integral}

\section{Beneficios de los programas de movilidad docente}

Los programas de movilidad profesoral en las Instituciones de Educación Superior han cobrado más sentido en la realidad de un mundo globalizado, por el hecho de aportar tanto a los docentes como a las instituciones participantes, una visión mucho más amplia para la efectividad de su función primordial, que es la generación de conocimientos (Bernal del Castillo, 2014; Castejón Silvo, 2013; Madarro, 2011).

La capacitación docente fuera del ámbito regular de funcionamiento, y el desarrollo de oportunidades para generar redes internacionales de investigación y docencia implica contribuir directamente en el logro de funciones que hoy en día son inherentes a la gestión docente. Todo tiene como propósito principal incidir en el proceso enseñanza aprendizaje para el logro de una mejora en la calidad académica (Knight, 2010).

Por su parte, la interculturalidad que se gesta como producto, enriquece cualquier proceso de investigación sobre las características de las distintas culturas, de su integración, vinculación y diversidad, aportando una visión más global de los problemas y soluciones, necesariamente conjuntas e integrales, que es un tema siempre presente en las agendas de los programas de educación en cualquier país.

Tal tipo de iniciativa propicia la internacionalización de las IES y estrecha lazos entre países, contribuye a aunar esfuerzos en la generación de un espacio común de educación superior, en el cual se ven beneficiadas cada una de las universidades participantes, no solo los docentes que forman parte del programa.

\section{El contexto de los Estudios Generales}

Los Estudios Generales constituyen el componente del currículo que enfatiza tanto en la importancia de las disciplinas como en su insuficiencia y limitaciones para la producción de conocimientos. También reconocen tanto la importancia de la razón, como los riesgos de un racionalismo a ultranza que ignora que los seres humanos, la subjetividad, la afectividad, y la vida, tienen un importante componente irracional. Al entender que las aptitudes cognitivas humanas solo pueden desarrollarse en el seno de una cultura que ha producido, conservado, $\mathrm{y}$ transmitido un lenguaje, una lógica; un conjunto de saberes y de criterios de verdad (Morin, 1999, p. 20), los Estudios Generales tienen una vocación integradora en la que se concibe al conocimiento como un fenómeno multidimensional-complejo que requiere de la conjunción de procesos 
energéticos, químicos, fisiológicos, cerebrales, existenciales, psicológicos, culturales, lingüísticos, lógicos, ideales, personales, inter y transpersonales y colectivos, los que se encuentran estrechamente vinculados y son inseparables unos de otros.

Los estudios generales reconocen la porosidad y vinculación de los saberes, así como la insuficiencia y limitación que representa la manera en que han sido organizados en las universidades (disciplinas, departamentos, facultades, carreras, etc.), sin dejar de valorar las aportaciones positivas que dicha organización ha aportado a la producción de conocimientos y capacitación de los estudiantes. De ahí que su principal aportación a la formación integral del estudiantado proviene de su énfasis en develar los fundamentos epistemológicos, económicos, políticos; en fin, culturales en sentido amplio, de todas las maneras de producir y organizar el conocimiento, tanto a través de la historia como en la actualidad. Así como la indisoluble vinculación de estos procesos con nuestra realidad y con las formas en que la representamos, construimos y reconstruimos, pero sobre todo experimentamos, disfrutamos, sufrimos y vivimos. En otras palabras, la educación general nos ayuda a restablecer la relación individuo-especie-sociedad, sin que esto conduzca a reducir o subordinar un término a otro. (Morin, 1993, pp. 22-23).

Los Estudios Generales, además -y tal vez, sobre todo- promueven activa y deliberadamente las conexiones entre las diversas áreas del saber, propiciando la reconstrucción de su tejido (complexus), así como el entendimiento de las razones históricas de su parcelación (disciplinarización) y los efectos que esto ha tenido y tiene en los procesos de aprendizaje y producción cultural. Además de caracterizarse por propiciar la conexión e integración de saberes provenientes de los diversos campos del conocimiento (ciencias sociales, ciencias naturales y humanidades), los Estudios Generales incorporan integralmente las experiencias y vivencias cotidianas de los estudiantes en los procesos de aprendizaje (Dewey, 2004;). Su pedagogía es plenamente dialógica, participativa, y democrática (Vélez Cardona, 2012).

Además, a partir de la literatura relevante se puede comprobar que los Estudios Generales han sido y son los principales responsables de promover la conciencia ciudadana en los estudiantes universitarios. Pero no una ciudadanía excluyente y con pretensiones homogeneizadoras, sino una ciudadanía intercultural y compleja (Tubino, 2003). En ese contexto, los Estudios generales promueven el desarrollo de la tolerancia, la empatía, la solidaridad y la afectividad; a la vez que ayudan a desarrollar competencias cognitivas e intelectuales de alto nivel, proveyendo las condiciones para la formación integral del estudiante (Vélez Cardona, 2012).

La formación integral que nos proveen los Estudios Generales será lo que mejor nos podrá ayudar, a los profesores, los estudiantes, así como otros integrantes del núcleo universitario a ubicarnos como actores sociales en todo el teatro de la vida, y a asumir plenamente nuestra ciudadanía de manera autónoma. Esto, en tensión y lucha abierta o encubierta, con un aparato publicitario que continuamente nos induce a ser espectadores de los otros y de nosotros (Freedman, 2009).

En la medida en que los Estudios Generales provocan una reflexión profunda sobre el papel que desempeña el ser humano, como ser biológico que produce y a la vez es producido (Morin, 2001) por reacciones químicas, sociales, fisiológicas, entre otras, en un espacio geofísico particular; es el componente curricular más capaz de propiciar el mejor entendimiento de nosotros mismos y de nuestras inter-retro relaciones con el entorno natural y social, lo que permitirá entendernos como sujetos sociales y solidarios comprometidos con el bienestar general de la población de nuestros países y planeta. Dicho de otro modo, es el componente del currículo universitario que mejor nos provee una formación integral (Vélez, Cardona, 2013). 


\section{Metodología y diseño}

Se realizó el diseño a partir de la siguiente hipótesis de trabajo:

La participación en los programas de movilidad docente, particularmente los que se desarrollan en el ámbito de los Estudios Generales tienen un impacto positivo y medible en el desarrollo de destrezas y actitudes de los estudiantes que conducen a su formación integral.

La operacionalización de las variables, destrezas, actitudes y formación integral, se realizó desagregando las dimensiones e indicadores a fin de identificar los ítems utilizados en las preguntas de los cuestionarios para docentes, así como para definir los criterios que fueron descritos en los instrumentos para autoevaluación, y el instrumento de observación de clases. Las dimensiones e indicadores pueden resumirse en áreas disciplinares, con intención de corroborar si el docente consideraba más de un área del saber, la integración de conocimiento y el establecimiento de importancia de esta integración, así también si proponía una metodología novedosa para abordar los problemas y situaciones que trata el curso en cuestión. También se consideró la incorporación de elementos históricos y filosóficos propios de la disciplina y otras áreas del saber. Esto se hizo siguiendo los criterios de una pedagogía basada en la docencia integradora y una evaluación auténtica, adecuadas para cursos de Estudios Generales.

La población estuvo compuesta por los seis docentes seleccionados, dos de cada país participante. Así también conforman la población los grupos de estudiantes de una sección de cada docente, seleccionada aleatoriamente.

La metodología consistió en establecer una triangulación entre las universidades participantes. El diseño fue mixto en el cual se utilizaron estrategias cualitativas y cuantitativas para la recogida y análisis de datos propios de ambos enfoques. Este tipo de diseño reconoce la triangulación de datos, fuentes de información, marcos teóricos y conceptuales, metodologías y perspectivas de los investigadores.

Con la información correspondiente al análisis y selección bibliográfica, se identificaron categorías y sub-categorías que dieron lugar a la construcción de los instrumentos que se utilizaron para la recolección de datos del pre-test y del pos-test.

Luego de los resultados de la primera etapa y con intención de dar profundidad a los datos recogidos, se diseñó otra entrevista para ser aplicada a los seis docentes participantes, dos de cada universidad; varios meses después de haber tenido la experiencia de movilidad.

En la primera etapa se hizo una revisión documental de los programas o fundamentaciones de estudios generales y de movilidad en cada universidad participante. Luego se construyeron los instrumentos para recoger la información (cuestionarios, entrevistas y rúbricas o matrices), para finalmente desarrollar las tablas y gráficas que sintetizan la información recopilada en matrices de categorías y subcategorías, así como los análisis que se derivan de ellas.

La recolección de información se realizó mediante varios instrumentos:

- Una guía de sistematización de datos de los programas de movilidad docente en cada universidad involucrada.

- Un cuestionario para las personas responsables de las unidades de movilidad profesoral en cada universidad.

- Un cuestionario para estudiantes de estudios generales en la UPR, en la PUCP y en INTEC.

- Entrevistas semi-estructuradas con los responsables de las áreas de Estudios Generales. 
- Rúbrica ${ }^{3}$ para observación de clases

- Rúbrica de autoevaluación docente

- Guía de entrevista para docentes

Los instrumentos utilizados fueron revisados para la aplicación del pos-test y solo el cuestionario a estudiantes tuvo un ajuste para mejor comprensión en una pregunta.

El diseño de la entrevista para docentes se realizó después de haber tenido la experiencia de la primera etapa de esta investigación.

\section{Análisis de resultados}

Los resultados obtenidos con todos los instrumentos aplicados reflejan diferencias en la práctica docente de los participantes luego de estar en el Programa de Movilidad. Las diferencias, en general, pueden agruparse en un mayor grado de dominio e incorporación de elementos pertinentes para la formación integral del estudiantado. El análisis de los resultados en esta etapa final, concluyó que sí existe un impacto directo positivo en la metodología, pedagogía, estrategias y recursos utilizados en la práctica de los docentes luego de participar en el Programa de Movilidad. Se refleja un enriquecimiento sobre todo en la integración de diversas áreas del saber y la referencia a distintos pensadores de diferentes culturas. También se manifiesta una mayor atribución de importancia a la integración de diversas áreas del saber en la docencia de los Estudios Generales.

Para analizar los datos en la interpretación y validación se utilizó Atlas.ti y teoría fundamentada, para la comprensión de la relación resultó adecuado la aplicación de redes semánticas y para la codificación y categorización se agruparon en las categorías identificadas.

3. El término rúbrica se refiere al conjunto de criterios y estándares relacionados con el aprendizaje.
La categoría original fue contrastada con la categoría emergente posterior a la movilidad, la manera de comparar modificaciones en métodos, contenido, transformación de prácticas, perspectiva didáctica, etc. Con intención de construir nuevas relaciones conceptuales y perspectivas que mejor apoyen la formación integral, las destrezas y actitudes en los aprendizajes.

A partir del análisis de los resultados, se podría condensar la conclusión tanto de estudiantes como docentes en la siguiente frase expresada por un docente:

"Todo fortalecimiento del docente repercute como un haz integral en el estudiante. Sin embargo, luego de este programa de Movilidad, lo más probable es que el nivel de incidencia en la formación integral se eleve con experiencias como la de co-docencia interactiva y presencial. Esta sacudida de la zona de confort que se le imprime a la educación tradicional, cuando se hacen irrumpir novedosas prácticas inclusivas, no tiene otro desenlace que la superación y la aspiración a la excelencia”.

En la segunda fase, autoevaluando los mismos elementos que en la primera, los docentes mostraron resultados distintos. Todos los docentes manifestaron cumplir con los aspectos relativos a la incorporación de diversas áreas del saber, integración del conocimiento, metodología y pedagogía. Los criterios de incorporación de elementos, delimitación de áreas del saber y la utilización de estrategias más adecuadas de evaluación fueron evaluados por uno de los docentes como área que necesita mejorar.

Partiendo de los resultados de las autoevaluaciones de la primera y segunda fase de la investigación, se evidencia que la percepción de los docentes sobre su práctica, antes y después del programa de movilidad, arroja diferencias. Los docentes luego de participar en el programa percibieron los elementos de los Estudios Generales más firmes dentro de su práctica docente. Esto podría indicar que luego 
de la participación en el Programa de Movilidad Docente, los participantes pudieron identificar en su práctica la incorporación de la concepción de los Estudios Generales de una manera más evidente. El cambio, la percepción estuvo ligada a la integración de distintas áreas del saber a través de una metodología y pedagogía integradora.

De los 166 estudiantes participantes, 158 contestaron que los docentes luego de la Movilidad, identificaban la asignatura como parte de los estudios generales y utilizaban estrategias integradoras con inclusión de muchas disciplinas.

Un ejemplo de la opinión de los docentes, la podemos identificar en uno de los profesores que indicó: "Si bien la asignatura era reconocida como de Estudios Generales, no incluía en su diseño de manera metódica actividades y técnica propias de otros enfoques y otras disciplinas, aún muy diferentes a las del área propia de mi asignatura”.

$\mathrm{Al}$ analizar los resultados de las observaciones docentes, en la primera fase, se observa que los elementos presentes en la mayoría de las prácticas docentes fueron la incorporación de elementos y la delimitación de las áreas del saber. A estos resultados le siguen la consideración de más de un área del saber, una metodología novedosa y una evaluación adecuada para un curso de estudios generales. Por último, los elementos que se observaron más débiles fueron la integración del conocimiento y una propuesta pedagógica y de docencia integradora.

En las observaciones de la segunda etapa se perciben cambios positivos. Todos los docentes mostraron considerar más de un área del saber, tener una propuesta metodológica novedosa para enfrentar las situaciones del curso, delimitar las áreas del saber y tener una pedagogía y práctica docente integradora. En los demás elementos la mayoría de los docentes mostraron integración del conocimiento, incorporación de elementos y evaluación adecuada para un curso de Estudios Generales. En estos últimos elementos sólo un docente mostró la necesidad de mejorar. Partiendo de la concepción de los Estudios Generales y la integración y reconstrucción de su complejidad, los resultados de las observaciones docentes sugieren un cambio, en este aspecto, a partir del Programa de Movilidad Docente. La incorporación de distintas áreas del saber, su delimitación y la precisión de su pertinencia se vieron presentes en las prácticas docentes. Además, el método de transmisión de los conocimientos y los contenidos utilizados consideraban la relación entre diversas disciplinas, diversas culturas y su utilidad en la materia específica del curso.

La participación en programas de Movilidad Docente es una experiencia enriquecedora que incide directamente en el desarrollo personal y profesional, estimula la creatividad, mejora las relaciones, y además fortalece el entendimiento multicultural. Este enriquecimiento se traduce a su vez en una concepción de enseñanza-aprendizaje que apunta a la formación integral de los estudiantes, volviéndose ésta un propósito inherente en sí mismo.

Durante la primera fase de la investigación los estudiantes participaron en un pre-test con ítems que evaluaban los elementos de Estudios Generales que los docentes incluían en sus clases, y la percepción de éstos sobre en qué medida eran implementados. Los resultados del pre-test mostraron como áreas fuertes la identificación de la asignatura como parte de los Estudios Generales, la comprensión de la diversidad de experiencias de sus estudiantes, la referencia a más de tres pensadores de otros países, y la comparación de la realidad del país con la de otros países. Los estudiantes calificaron en un nivel medio los aspectos relacionados con la integración de conocimientos de otras disciplinas y la incorporación de experiencias del estudiantado en el día a día de los cursos. La evaluación más baja que hicieron fue sobre la referencia a diversas modalidades de estudios generales y la asignación de ejercicios y/o tareas para que incorporen la realidad (presente o pasada) de otros países. 
Los resultados del pre-test muestran una identificación de la asignatura dentro de los Estudios Generales y la integración cultural de los conocimientos, así como la comprensión de la diversidad de experiencia de los estudiantes dentro del proceso de enseñanza-aprendizaje. Estos aspectos apuntan en la dirección de los Estudios Generales, aunque en el momento carecen de firmeza elementos fundamentales como la integración de conocimientos de otras disciplinas, la incorporación de las experiencias del estudiantado en la cotidianidad de la clase y asignaciones de tareas que incorporen la realidad (presente o pasada) de otros países.

En la segunda etapa se administró el mismo instrumento a modo de post-test. Estos resultados mostraron algunas diferencias. Las áreas más fuertes relacionadas con Estudios Generales fueron la identificación de la asignatura como parte de los Estudios Generales, la referencia a más de tres pensadores o teóricos de otros países, la comparación de la realidad del país con la de otros países y la referencia a las diversas modalidades de Estudios Generales.

La incorporación de las experiencias del estudiantado en las clases fue evaluada en un nivel medio, así como la integración de conocimientos de otras disciplinas o áreas del saber, diferentes a las del curso. Además de caracterizarse por propiciar la conexión e integración de saberes provenientes de los diversos campos del conocimiento (ciencias sociales, ciencias naturales y humanidades) los Estudios Generales incorporan integralmente las experiencias y vivencias cotidianas de los estudiantes en los procesos de aprendizaje (Dewey, 2004). Su pedagogía es plenamente dialógica, participativa y democrática. Estos aspectos, aunque en nivel medio, fueron percibidos por los estudiantes en las prácticas de los docentes luego de su participación en el programa de Movilidad. La evaluación más baja estuvo relacionada con la asignación de ejercicios y/o tareas para que incorporen la realidad (presente o pasada) de otros países.
Entre los distintos resultados de la primera y segunda etapa se pueden notar cambios en distintos aspectos relativos a los Estudios Generales, antes y después de la participación en el programa de Movilidad Docente. Sobre todo, en aspectos como la integración de distintas áreas del saber y la pedagogía práctica docente integradora. Estos cambios conllevan una metodología y práctica docente que se fundamenta en la complejidad del conocimiento y en la interacción de los saberes más allá de las disciplinas, generando un aprendizaje holístico que considera todas las ramas del saber que por su naturaleza se involucran en la asignatura que se imparte. Además de adoptar esta concepción pedagógica y ponerla en práctica, los docentes mostraron inclinación a integrar la experiencia de los estudiantes y a considerarlos como parte activa del proceso de enseñanza-aprendizaje.

A modo de ejemplo, podemos señalar a un estudiante que indicó: "La profe siempre nos escucha y nos hace participar en clases, pero ahora [refiriéndose con posterioridad a la participación en el programa de Movilidad] no es ella la que dirige la pregunta, sino que deja que seamos nosotros quienes expliquemos sobre nuestras formas de entender nuestros problemas. Es como si ahora incluyera nuestras inquietudes como parte de la materia.

Como se ha establecido anteriormente, los Estudios Generales se fundamentan en la integración: de conocimientos, culturas, historias, metodologías didácticas y contextos del estudiante. La experiencia de capacitación a través del programa de Movilidad brindó a los docentes la oportunidad de desarrollar herramientas que le permitieran integrar todos estos aspectos, convirtiéndose en un factor fundamental en la formación integral de los estudiantes.

Para romper con la fragmentación de las disciplinas es necesario integrar el proceso docente -educativo, lo que implica la sistematización paulatina de los contenidos y de sus relaciones, de manera que estos se vayan acercando cada vez más a la 
realidad circundante y compleja que nos presenta la vida. Según los resultados obtenidos, en los distintos instrumentos aplicados, luego del proceso de Movilidad Docente, las clases incluyeron elementos que se acercan a este proceso.

La disciplina con concepción integradora debe servir de enlace con el resto de las disciplinas. A partir de la resolución de problemas donde el estudiante realiza abstracciones de la realidad, forma su pensamiento y desarrolla habilidades propias para el estudio y trabajo con los contenidos de las ciencias o ramas del saber vinculados a su profesión, ya que esas habilidades se incorporan a la solución de problemas de las disciplinas integradoras, que son, en última instancia, los de la realidad social misma (Ferreira, 2004). El espacio de poder contrastar las realidades brinda la oportunidad de desarrollar habilidades generalizadoras que se corresponden a las desarrolladas en otras disciplinas. Cuando los estudiantes son expuestos a una formación integradora como la que demostraron los docentes participantes de la investigación, se favorece el desarrollo de conexiones entre los saberes y es posible que los egresados sean capaces de desenvolverse tanto en su ámbito profesional, como en la sociedad. Esto se debe a que la metodología de enseñanza-aprendizaje, de la que son parte activa, vincula la realidad social, económica, cultural, etc. al entorno que les rodea.

\section{Conclusiones y recomendaciones}

Es preciso destacar que la duración de la estancia de los docentes en las universidades en las que compartieron docencia, fue de apenas una semana. Sin embargo, esta fue muy intensa, sobre todo por la diversidad de actividades en las que participaron: docencia, co-docencia, conferencias, seminarios de capacitación docente, seminarios para estudiantes, visita a clases de docentes de varias áreas del saber, diálogos formales e informales con grupos de docentes, con grupos de estudiantes, con administradores, actividades artísticas y culturales, entre otras.

A continuación se presentan las conclusiones para cada uno de los objetivos que nos trazamos en esta etapa de la investigación.

\section{Objetivo 4: Describir de qué manera se ven modificadas las prácticas pedagógicas a partir de la participación en el programa}

En los resultados de la etapa final de esta investigación se evidencian modificaciones en las prácticas pedagógicas luego de la participación en el programa de Movilidad Docente. Según los resultados de las autoevaluaciones y las observaciones docentes las prácticas docentes incluyeron más elementos de integración después de la participación en el programa.

Los cambios fueron identificados a nivel de contenido y de la relación de estos dentro de diversas áreas del saber. Las prácticas pedagógicas incluían más áreas del saber que, de distintas maneras, se relacionaban con los contenidos del curso. Además, los docentes hacían una conexión explícita entre la esencia de la asignatura y las distintas disciplinas con las que se relacionan. Al hacer esta integración de saberes los docentes también se ocupaban de establecer la importancia de la misma.

Las prácticas pedagógicas luego de la participación en el programa, apuntaban a dar respuestas a los problemas de la clase partiendo de la delimitación de las áreas del saber pertinentes. Otro elemento que sufrió modificaciones fue la evaluación, ya que luego del programa los docentes implementaron metodologías de evaluación más ajustadas a los Estudios Generales.

Las prácticas pedagógicas también mostraron incluir más referencias a la historia de otros países y culturas, así como el uso de pensadores provenientes de diferentes países. A la vez este elemento 
de la práctica docente incluye el contraste de los acontecimientos pasados y los presentes.

En general, las prácticas pedagógicas luego de la participación en el programa de Movilidad Docente, se ven modificadas en la metodología de enseñanza-aprendizaje, el contenido, las actividades prácticas y la evaluación. En cada aspecto se incluye una visión integral de aprendizaje que comprende distintas áreas del saber y su relación con la asignatura impartida.

\section{Objetivo 5: Evaluar si existen modificaciones en las estrategias de formación integral a partir de la participación en el programa}

Partiendo de los resultados de la etapa final de la investigación, se pueden identificar modificaciones en las estrategias de formación integral de los docentes. Las modificaciones se ven reflejadas en el abordaje hecho por los docentes luego de participar en el programa de Movilidad Docente.

De esta forma, a través de las estrategias de formación integral, los estudiantes fueron expuestos a diferentes realidades que permitieron el contraste de los saberes a nivel de las disciplinas y en ocasiones a nivel cultural. Este tipo de análisis impacta la forma de aprender de los estudiantes, les invita a poner en práctica el pensamiento reflexivo y crítico. El aprendizaje generado a través de esta experiencia es transferible a distintas áreas de la vida cotidiana, abriendo de esta forma la formación holística del estudiante, que impacta todos los aspectos de su vida más allá del área profesional.

La integración de los saberes a través de una metodología y pedagogía integral desarrolla habilidades en los estudiantes que les permite hacer conexiones entre las disciplinas para comprender y solucionar los problemas. Además, les brinda amplitud de conocimientos respecto a diferentes países.

Las habilidades generables como se mencionó anteriormente son transferibles a distintas áreas, es por esto que tienen un gran valor para el desarrollo personal y profesional de los estudiantes. La formación integral permite el desarrollo de estas habilidades que a su vez ayuda a los estudiantes a explorar distintas áreas del saber para armar la complejidad del pensamiento. La capacidad de abstracción y construcción del conocimiento partiendo de las conexiones hechas entre los distintos elementos que interactúan en una asignatura, desarrollan la capacidad creativa y en gran medida la identificación y solución de problemas. Además, despierta y desarrolla el espíritu investigativo que permite la generación de más conocimiento.

\section{Objetivo 6: Evaluar la percepción de estudiantes respecto a las estrategias utilizadas por los docentes que participan en el programa de movilidad}

Uno de los propósitos de esta investigación consistió en recoger información acerca de la percepción de los estudiantes sobre las prácticas docentes de los participantes. Sobre este particular se evaluaron aspectos como la identificación de la asignatura, por parte del docente, como parte de los Estudios Generales, la referencia a pensadores o teóricos provenientes de otras culturas o países, la comparación de la realidad del país con la de otros, la incorporación o integración de conocimientos de otras disciplinas o áreas del saber, diferentes a las del curso, la asignación de actividades que incorporan la realidad presente y pasada de otros países, la sensibilidad ante la diversidad de experiencia de sus estudiantes, incorporación de las experiencias del estudiantado en el día a día de los cursos y la referencia a diversas modalidades de Estudios Generales. Cada uno de estos aspectos fue evaluado de acuerdo al grado de incorporación y/o la cantidad de elementos incorporados.

Los resultados sugieren una diferencia en la percepción de los estudiantes antes y después de la 
participación de los docentes en el programa de Movilidad. Los estudiantes percibieron que luego del programa los docentes identificaron más la asignatura como parte de los Estudios Generales. También manifestaron que se hacía mayor referencia al menos a más de tres pensadores o teóricos de otros países, más comparación con la realidad de otros países y más asignación de ejercicios incorporando la realidad de (presente o pasada) de otros países. Por otro lado, los estudiantes percibieron que luego de la participación en el programa, los docentes se mostraban más sensibles y comprensivos a la diversidad de experiencias de los mismos y a la vez haciendo más integración de estas experiencias del estudiantado en el día a día de la clase. Otro aspecto importante de la percepción fue la mayor referencia a diversas modalidades de Estudios Generales.

Tomando como referencia los resultados de la percepción de los estudiantes en los distintos aspectos mencionados, se hace evidente que para ellos, luego de la participación de los docentes en el programa de Movilidad, las prácticas docentes estaban más orientadas a la formación integral a través de los Estudios Generales. Las percepciones describen prácticas pedagógicas integradoras que incorporan diferentes saberes y realidades en el proceso de enseñanza-aprendizaje de una asignatura en Estudios Generales.

Sobre la pregunta abierta aplicada en el post-test sobre cuál entienden los estudiantes que es la modalidad más apropiada para su Universidad, las respuestas fueron variadas, pero apuntando en una misma dirección. Las respuestas fueron categorizadas de la siguiente manera:

- Una modalidad interactiva y participativa donde docente y estudiantes se relacionen.

- Modalidad dinámica con énfasis en la investigación.

- Modalidad Holística con estudios inter y transdisciplinarios de los diferentes cursos.
- Una modalidad que contraste la clase con el contexto real.

Si se toma en consideración esta categorización de las respuestas de los estudiantes acerca de lo que ellos consideran una modalidad apropiada para su Universidad y se contrasta con los resultados obtenidos en el post-test sobre sus percepciones acerca de las prácticas docentes, se puede ver cómo estas incluyen la mayoría de los elementos que el estudiantado considera importante. Cabe destacar que estas características de formación integral fueron observadas después de la experiencia de los docentes en el programa de Movilidad, lo cual sugiere que el impacto del programa afecta directamente la práctica de los docentes a favor de una propuesta pedagógica y metodología más integral.

Los resultados sobre cómo se ven modificadas las prácticas pedagógicas de los docentes, la modificación en la formación integral de los estudiantes y la percepción de los estudiantes sobre las prácticas docentes antes y después de la participación en el programa de Movilidad sugieren que hay un cambio positivo en las estrategias docentes y por ende en la formación de los estudiantes.

Los resultados obtenidos, así como el entusiasmo generado por el Programa de Movilidad Docente en Estudios Generales, sirve de aval para recomendar que esta experiencia se amplíe, tanto en términos de extensión de las estancias como en la incorporación de más universidades $y$, sobre todo, en la cantidad de docentes que participen de esta experiencia. Existe un gran consenso en la comunidad de educación superior internacional en que la capacitación de los docentes es de fundamental importación para el funcionamiento de las universidades y, sobre todo, para la formación intelectual del estudiantado.

En el caso de los Estudios Generales, principales responsables de aportar a la formación integral de los estudiantes, la capacitación de los docentes es mucho más importante aún. Particularmente 
porque estos llegan a las aulas con una formación especializada en una disciplina y se ven precisados a ampliar sus miras y considerar diversas dimensiones de la realidad al ejercer su docencia. Es por ello que un programa de Movilidad como el que hemos estado evaluando en esta investigación resulta de extraordinaria importancia para aquellas instituciones que tienen el compromiso de brindarle una formación integral a los estudiantes que acuden a sus aulas. Por eso consideramos que debe darse continuidad y ampliarlo, comprendiendo la gran importancia en la formación integral del estudiantado, que este programa ha evidenciado.

\section{Referencias}

Bernal del Castillo, J. (2014, junio). El proyecto de intercambio de movilidad docente Erasmus en el ámbito delas ciencias penales. Revista jurídica de investigación e innovación educativa, (10), 127-140.

Castejón Silvo, N. (2013). Programas de movilidad en CUD: Panorámica evaluativa general. En Irisarri Primicia, S. (Coord.) Evaluación de Programas de Movilidad en Cooperación Universitaria para el Desarrollo (pp. 47-56). Pamplona: Universidad Pública de Navarra.

Córdoba, M. \& Vélez, W. (2015). El reto de la movilidad docente y los estudios generales: un estudio en dos etapas. Ciencia y Sociedad, 40(4), 725-744.

Dewey, J. (2004). Democracia y educación (sexta edición). Buenos Aires: Losada.

Ferreira, G. (2004) Hacia la integración curricular en la Educación Superior: reflexiones, necesidades y propuesta para la disciplina integradora. Revista Iberoamericana de Educación. 34(2). Recuperado de: http://rieoei.org/edu_sup33.htm

Freedman, R. (2009). Noise Wars. Compulsory Media and Our Loss of Autonomy. New York: Algora Publishing.
Kelle, U. (2006). Combining Qualitative and Quantitative Methods in Research Practice: Purposes and Advantages. Qualitative Research in Psychology, (3), 293-311.

Knight, J. (2010). Internacionalización de la Educación Superior. Toronto, Canadá: Instituto de Ontario de Estudios en Educación. Universidad de Toronto.

Instituto Tecnológico de Santo Domingo. (2017). Estrategia 2017. Santo Domingo: INTEC.

Madarro, A. (2011). Redes de movilidad académica para la cooperación e integración regional en Iberoamérica. Revista Iberoamericana de Educación, (57) 71-107

Marsh, C. \& Willis, G. (2007). Curriculum: Alternative Approaches, Ongoing Issues (4ta. ed.) Upper Saddle River, New Jersey: Pearson y Merrill Prentice Hall.

Martin, P. (2009, May). Study Abroad Programs: Elements of Effective International Students and Faculty Exchange Programs. Sacramento, California: California Research Bureau.

Martínez, M., Ríos E. \& Vélez, W. (2012). La investigación institucional en las instituciones de Educación Superior en Puerto Rico: Una tipología de la Oficina de Investigación Institucional. Puerto Rico: CEDESP.

Mercado Común del Sur. (2015). Plan estratégico del sector educativo. 2011-2015.

Ministerio de Educación Argentino. Programa de Internacionalización de la Educación Superior y Cooperación Internacional (PIESCI). Recuperado de educacion.gov.ar/spu/cooperacion-internacional

Morin, E. (1993). El método I: La naturaleza de la naturaleza. Madrid: Ediciones Cátedra.

Morin, E. (1999). El método III: El conocimiento del conocimiento. Madrid: Ediciones Cátedra.

Morin, E. (2001). El método IV: Las ideas. Madrid: Ediciones Cátedra. 
Morin, E. (2003). Introducción al pensamiento complejo. Barcelona: Gedisa Editorial. Asunción: MECASUR.

Nicolescu, B. (2002). Manifesto of Transdisciplinarity. Albany, NY: State University of New York Press.

Organización de Estados Iberoamericanos para la Educación, la Ciencia y la Cultura. (2015). Redes académicas de Movilidad Docente. Un intercambio de experiencias iberoaméricanas para conocer diversas realidades desde la práctica. Madrid: España: Organización de Estados Iberoamericanos para la Educación, la Ciencia y la Cultura (OEI).

Organización de Estados Iberoamericanos para la Educación, la Ciencia y la Cultura. (2012). Informe Anual del Programa Pablo Neruda. Madrid, España: Organización de Estados Iberoamericanos para la Educación, la Ciencia y la Cultura (OEI), Unidad Técnica Pablo Neruda.

Pontifica Universidad Católica del Perú. (2017). Estrategia 2017. Lima: Pontifica Universidad Católica del Perú.

Quero, S. (2008). Estrategias integradoras para el abordaje de la educación integral. Maracaibo, Venezuela: Universidad del Zulia.

Sebastián, J. (2002). Oportunidades e iniciativas para la cooperación iberoamericana en educación superior. Revista Iberoamericana de educación, (28),197225. Sebastián, J. (2004). Cooperación e internacionalización de las universidades. Buenos Aires: Biblos.

Sisco, L. A. (2007, Sept.). Learning to See What's Invisible: The Value of International Faculty Exchange. Business Education Quarterly, 354-360.

Siufi, G. (2009, enero). Cooperación internacional e internacionalización de la educación superior. Nueva Época, 123-145.
Subirats, P. \& Vélez, W. (2010). La educación general en el Siglo XXI. Ponencia presentada en el Primer Simposio Internacional de Estudios Generales. San Juan, Puerto Rico: Universidad de Puerto Rico, Recinto de Río Piedras, 21p.

Tiana, A. (2012). La contribución de la movilidad académica a la construcción de un espacio iberoamericano de educación superior. Revista Lusófona de Educação (21), 53-68.

Tubino, F. (2003). Ciudadanías complejas y diversidad cultural. En Vigil, N. \& Zariquiey, R. (Eds.). Ciudadanias inconclusas. El ejercicio de los derechos en sociedades asimétricas (pp.167-191). Lima, Perú: Cooperación Alemana al Desarrollo y PUCP.

Universidad Católica de Córdoba. (2008). Jornadas Docentes. Córdoba: Universidad Católica de Córdoba.

Universidad de Puerto Rico. Estrategia institucional 2016. San Juan, PR: Universidad de Puerto Rico.

Volkwein, J. F. (2008). The Foundations and Evolution of Institutional Research. New Directions for Higher Education, No. 141, pp. 5-20.

Vélez, W. \& Subirats, P. (2014). Apuntes para un perfil del profesor de Educación General. Cuaderno de Pedagogía Universitaria, 7(14), 17-20.

Vélez Cardona, W. (2013). Integración de saberes y formación integral en los estudios generales del Siglo XXI. Memorias del V Simposio Internacional de Estudios Generales. Recuperdado de: www.rideg. org/memorias/v-simposio/, $7 \mathrm{p}$.

Vélez Cardona, W. (2012). Educación general: definiciones, evolución, paradigmas epistemológicos y futuro. Río Piedras, PR: Facultad de Estudios Generales, Universidad de Puerto Rico. 


\section{Datos de filiación}

Dra. María Elena Córdoba. Educadora investigadora. Estudió Psicología en la Universidad Nacional de Córdoba, Argentina, cursó una Maestría en Educación Social y Animación Sociocultural en la Universidad de Sevilla y un Doctorado en Educación en el Atlantic International University. En el campo académico ocupó cargos en la Secretaría de Educación y en reconocidas Universidades de México. Actualmente se desempeña como docente y consultora en Educación. Su línea principal de investigación son los estudios generales y la transdiciplinariedad. Correo electrónico: macorbu@yahoo.com

Dr. Waldemiro Vélez. Catedrático del Departamento de Ciencias Sociales, de la Facultad de Estudios Generales, Recinto Río Piedras de la Universidad de Puerto Rico. Coordinador de la Red Internacional de Estudios Generales (RIDEG). Correo electrónico: waldemirov@hotmail.com 